\title{
High-Pressure Melting Experiments on Basalt-Peridotite Layered Source (KLB-1/N-MORB): Implications for Magma Genesis in Hawaii
}

\author{
Shan Gao' , Eiichi Takahashi' ${ }^{1,2}$, Toshihiro Suzuki ${ }^{1}$ \\ ${ }^{1}$ Magma Factory, Earth and Planetary Science Department, Tokyo Institute of Technology, Tokyo, Japan \\ ${ }^{2}$ Guangzhou Institute of Geochemistry, Chinese Academy of Science, Guangzhou, China \\ Email: *etakahas@geo.titech.ac.jp, *takahashi@gig.ac.cn
}

How to cite this paper: Gao, S., Takahashi, E. and Suzuki, T. (2017) High-Pressure Melting Experiments on Basalt-Peridotite Layered Source (KLB-1/N-MORB): Implications for Magma Genesis in Hawaii. International Journal of Geosciences, 8, 1-15. http://dx.doi.org/10.4236/ijg.2017.81001

Received: December 7, 2016

Accepted: January 13, 2017

Published: January 16, 2017

Copyright $\odot 2017$ by authors and Scientific Research Publishing Inc. This work is licensed under the Creative Commons Attribution International License (CC BY 4.0).

http://creativecommons.org/licenses/by/4.0/

\begin{abstract}
In order to understand the melting processes that occur within recycled oceanic crust and mantle in a heterogeneous plume (e.g., that beneath the Hawaiian Islands), a series of high-pressure-high-temperature layered experiments were performed at $2.9 \mathrm{GPa}, 5 \mathrm{GPa}$, and $8 \mathrm{GPa}$, from $1300^{\circ} \mathrm{C}$ to $1650^{\circ} \mathrm{C}$, using a fertile peridotite KLB- 1 and N-MORB. Our experiments at conditions below the dry peridotite solidus produced melt compositions that ranged from basaltic andesite to tholeiite. An Opx reaction band formed between eclogite and peridotite layers, likely via chemical reaction between a silica-rich eclogite-derived partial melt and olivine in the peridotite matrix. At temperatures at or above the dry peridotite solidus, substantial melting occurred in both basalt and peridotite layers, and fully molten basalt melt and melt pockets from the peridotite layer combined. In our layered experiments, major and minor element contents in reacted melts closely matched those of Hawaiian tholeiite and picrite, except for Fe. Partial melts of anhydrous run products had $\sim 55-57 \mathrm{wt} \% \mathrm{SiO}_{2}$ at low temperature (i.e., were andesitic) and had $\sim 50$ $53 \mathrm{wt} \% \mathrm{SiO}_{2}$ at high temperatures, slightly below the dry peridotite solidus (i.e., were tholeiitic, and similar to those that occur during the Hawaii shield-building stage). Based on the Fe- and LREE-enriched signature in Hawaiian tholeiites, we propose that recycled components in the Hawaiian plume are not modern N-MORB, but are Fe-rich tholeiite; a lithology that was common in the Archaean and early Proterozoic. We have demonstrated that the entire compositional spectrum of Hawaiian tholeiites (basalt to picrite) can be formed by basalt-peridotite reactive melting near the dry solidus of peridotite. Based on these results, we propose that the potential temperature of the sub-Hawaiian plume may be much lower than previously estimated.
\end{abstract}




\section{Keywords}

Hawaiian Tholeiite, Recycled Oceanic Crust, High Pressure, Layered Experiments, N-MORB, Major Elements

\section{Introduction}

The mantle plume beneath the Hawaiian Islands is the largest known example in the Earth today [1]. Unlike other ocean islands that are dominated by alkali basalts, more than $90 \%$ of the volcanic products on Hawaii are tholeiitic in composition [2]. The Kilauea volcano is currently in its shield-building stage, whereas the Mauna Loa volcano will imminently end its shield-building stage. These Hawaiian tholeiites were once thought to be the products of melting of upper-mantle peridotite at extreme temperatures [3], although such peridotite-derived magmas are lower in $\mathrm{SiO}_{2}, \mathrm{FeO}, \mathrm{TiO}_{2}$, and $\mathrm{K}_{2} \mathrm{O}$ than Hawaiian tholeiite for any given $\mathrm{MgO}$ content [4] [5]. It is now generally accepted that the tholeiite lavas that contribute to the main shield-building stage are supplied by reactive melting of peridotite and mafic lithologies entrained in the plume axis [6]-[11]. Dynamic modelling of eclogite-peridotite hybrid plumes has accounted for the distribution of Kea-type and Loa-type volcanoes, as well as the sequential change of igneous rock types in shield volcanoes [12] [13]. Further, several melting experiments and numerical modelling simulations of heterogeneous mantle plumes have been performed recently in order to clarify the petrogenesis of tholeiite magma in Hawaiian plume [7] [14] [15] [16].

Sobolev et al. [10] proposed that Hawaiian tholeiite magma is formed from melting of a pyroxenite source rock. For this mechanism, they suggested that olivine in mantle peridotite is consumed by reaction with silicic melts derived from deep-seated partial melting of recycled oceanic crust, which thus forms a secondary pyroxenite source. Mallik and Dasgupta [17] showed experimentally that a wall-rock reaction between MORB-derived eclogite and peridotite could produce magmas with compositions from tholeiitic basalt to alkali basalt; however, the melting processes that may occur in such a hybrid source are disputed. In this study, we carried out a series of high-temperature-high-pressure melting experiments in order to elucidate the physical and geochemical characteristics of melting processes in layered basalt-peridotite source. Layered experiments were carried out at a pressure of $2.9 \mathrm{GPa}$ using a piston-cylinder apparatus, and at 5 $\mathrm{GPa}$ and $8 \mathrm{GPa}$ using a multi-anvil apparatus. A fertile spinel-lherzolite and $\mathrm{N}$-type mid-ocean ridge basalt (MORB) were used as starting materials.

\section{Experimental Approach}

\subsection{Starting Materials}

N-type MORB sample NAM-7 [18] was used as the eclogite component in our experiments, whereas spinel lherzolite sample KLB-1 was used as the fertile 
mantle peridotite component [4]. The chemical compositions and representative mineral constituents of each starting material are presented in Table 1. Both rock types were powdered and dried at 1 atmosphere under a $\mathrm{CO}-\mathrm{H}_{2}$ buffer, which is close to an NNO buffer, and were kept in a furnace at $200^{\circ} \mathrm{C}$ in order to prevent atmospheric hydration.

\subsection{Experimental Conditions}

Our melting experiments were performed at pressures of $2.9 \mathrm{GPa}, 5 \mathrm{GPa}$, and 8 $\mathrm{GPa}$, temperatures from $1300^{\circ} \mathrm{C}$ to $1650^{\circ} \mathrm{C}$, and for durations of $1-24$ hours (Figure 1 and Table 2). All run conditions and the resultant phases produced are listed in Table 2. Anhydrous solidi for KLB-1 and N-MORB NAM-7 are shown in Figure 1 (after [4] and [18], respectively), where it can be seen that the latter is situated at temperatures $\sim 200^{\circ} \mathrm{C}$ below than that of the former. In order to investigate wall-rock reactions between molten eclogite and ambient mantle peridotite in an ascending plume, most of the experiments in this study were conducted at temperatures below the dry peridotite solidus.

\subsection{Experimental Apparatus and Sample Assembly}

All experiments were carried out at the Magma Factory in the Tokyo Institute of Technology, Japan [19]. Each individual rock powder was pressed into a separate pellet, and both were then placed into graphite/platinum double capsules to avoid Fe-loss. Figure 2 shows the capsule assembly with the N-MORB layer placed on the top of peridotite layer near to the thermocouple.

Table 1. Chemical compositions of starting materials.

\begin{tabular}{|c|c|c|c|c|c|c|}
\hline & \multicolumn{5}{|c|}{ KLB-1 } & \multirow{2}{*}{$\begin{array}{c}\text { NAM-7 } \\
(w t \%)\end{array}$} \\
\hline & Bulk (wt\%) & $\begin{array}{c}\text { Oliv } \\
\text { (wt\%) }\end{array}$ & $\begin{array}{c}\text { Opx } \\
(\mathrm{wt} \%)\end{array}$ & $\begin{array}{c}\mathrm{Cpx} \\
(\mathrm{wt} \%)\end{array}$ & Sp (wt\%) & \\
\hline $\mathrm{SiO}_{2}$ & 44.48 & 39.64 & 54.24 & 51.13 & 0.06 & 49.71 \\
\hline $\mathrm{TiO}_{2}$ & 0.16 & 0 & 0.11 & 0.58 & 0.11 & 1.71 \\
\hline $\mathrm{Al}_{2} \mathrm{O}_{3}$ & 3.59 & 0.03 & 4.97 & 7.4 & 58.48 & 15.68 \\
\hline $\mathrm{FeO}(\mathrm{t})$ & 8.1 & 10.52 & 6.57 & 3.11 & 10.68 & 9.46 \\
\hline $\mathrm{MnO}$ & 0.12 & 0.16 & 0.16 & 0.1 & -- & 0.18 \\
\hline $\mathrm{MgO}$ & 39.22 & 48.25 & 32.16 & 14.7 & 21.61 & 8.43 \\
\hline $\mathrm{CaO}$ & 3.44 & 0.08 & 0.85 & 19.54 & 0 & 11.73 \\
\hline $\mathrm{Na}_{2} \mathrm{O}$ & 0.3 & 0.01 & 0.12 & 1.72 & 0 & 2.76 \\
\hline $\mathrm{K}_{2} \mathrm{O}$ & 0.02 & 0 & 0 & 0.01 & 0 & 0.23 \\
\hline $\mathrm{P}_{2} \mathrm{O}_{5}$ & 0.03 & - & - & - & - & 0.02 \\
\hline $\mathrm{Cr}_{2} \mathrm{O}_{3}$ & 0.31 & 0.01 & 0.34 & 0.78 & 7.82 & - \\
\hline $\mathrm{NiO}$ & 0.25 & 0.39 & 0.11 & 0.11 & 0.43 & - \\
\hline Total & 100.02 & 99.09 & 99.63 & 99.18 & 99.19 & 99.91 \\
\hline $\mathrm{MgO \#}$ & 0.896 & 0.891 & 0.897 & 0.894 & 0.783 & 0.614 \\
\hline
\end{tabular}




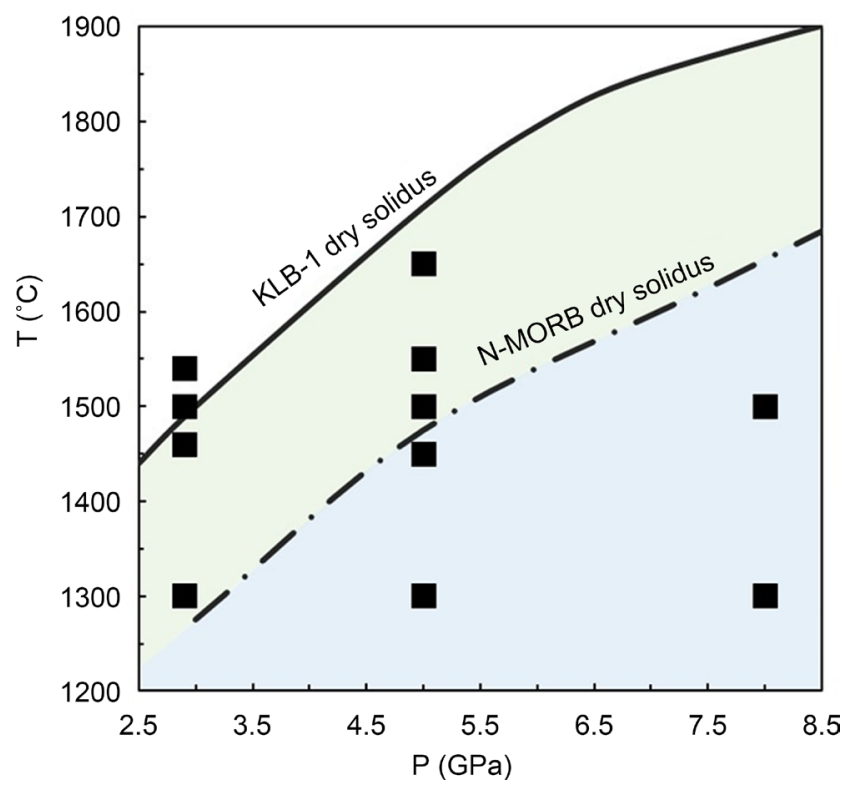

Figure 1. Solidus of peridotite KLB-1, NMORB and run conditions.

Table 2. Run conditions and phase assemblages of each layer.

\begin{tabular}{|c|c|c|c|c|c|}
\hline \multirow{2}{*}{ Run No. } & \multirow{2}{*}{$\mathrm{P} / \mathrm{GPa}$} & \multirow{2}{*}{$\mathrm{T} /{ }^{\circ} \mathrm{C}$} & \multirow{2}{*}{ Durations/h } & \multicolumn{2}{|c|}{ Mineral Modes (Weight Fraction) } \\
\hline & & & & MORB Layer & KLB-1 Layer \\
\hline P948 & 2.9 & 1300 & 3 & $\mathrm{Cpx}+\mathrm{Pl}+\mathrm{Grt}+\mathrm{Rt}$ & $\mathrm{Ol}+\mathrm{Opx}+\mathrm{Cpx}+\mathrm{Sp}+\mathrm{Grt}(\operatorname{tr})$ \\
\hline P951 & 2.9 & 1300 & 24 & $\mathrm{Cpx}+\mathrm{Grt}+\mathrm{Coe}$ & $\mathrm{Ol}+\mathrm{Opx}+\mathrm{Cpx}+\mathrm{Grt}(\operatorname{tr})$ \\
\hline P928 & 2.9 & 1460 & 1 & $\mathrm{Cpx}+\mathrm{Grt}+\mathrm{M}$ & $\mathrm{Ol}+\mathrm{Opx}+\mathrm{Cpx}+\mathrm{Sp}+\mathrm{Grt}(\mathrm{tr})$ \\
\hline P937 & 2.9 & 1460 & 9 & M & $\mathrm{Ol}+\mathrm{Opx}+\mathrm{Cpx}+\mathrm{M}(\operatorname{tr})$ \\
\hline P931 & 2.9 & 1460 & 24 & $\mathrm{Cpx}+\mathrm{Grt}+\mathrm{M}$ & $\mathrm{Ol}+\mathrm{Opx}+\mathrm{Sp}+\mathrm{Grt}(\mathrm{tr})$ \\
\hline P929 & 2.9 & 1500 & 1 & $\mathrm{Cpx}+\mathrm{Grt}+\mathrm{M}$ & $\mathrm{Ol}+\mathrm{Opx}+\mathrm{Cpx}+\mathrm{Sp}+\mathrm{Grt}(\operatorname{tr})$ \\
\hline P938 & 2.9 & 1500 & 9 & M & $\mathrm{Ol}+\mathrm{Opx}+\mathrm{M}$ \\
\hline P935 & 2.9 & 1500 & 24 & $\mathrm{Cpx}+\mathrm{Grt}+\mathrm{M}$ & $\mathrm{Ol}+\mathrm{Opx}+\mathrm{Cpx}$ \\
\hline P932 & 2.9 & 1540 & 1 & $\mathrm{Cpx}+\mathrm{M}$ & $\mathrm{Ol}+\mathrm{Opx}+\mathrm{Cpx}+\mathrm{Grt}+\mathrm{M}(\mathrm{tr})$ \\
\hline P939 & 2.9 & 1540 & 9 & M & $\mathrm{Ol}+\mathrm{Opx}+\mathrm{Cpx}+\mathrm{M}$ \\
\hline P936 & 2.9 & 1540 & 24 & M & $\mathrm{Ol}$ \\
\hline S1813 & 5 & 1300 & 3 & $\mathrm{Cpx}+\mathrm{Grt}+\mathrm{Coe}$ & $\mathrm{Ol}+\mathrm{Opx}+\mathrm{Cpx}+\mathrm{Grt}(\mathrm{tr})+\mathrm{Sp}(\operatorname{tr})$ \\
\hline S1797 & 5 & 1450 & 3 & $\mathrm{Cpx}+\mathrm{Grt}+\mathrm{M}$ & $\mathrm{Ol}+\mathrm{Cpx}+\mathrm{Opx}+\mathrm{Grt}+\mathrm{Sp}(\mathrm{tr})$ \\
\hline S1802 & 5 & 1450 & 20 & $\mathrm{Cpx}+\mathrm{Grt}+\mathrm{M}$ & $\mathrm{Ol}+\mathrm{Cpx}+\mathrm{Opx}+\mathrm{Grt}+\mathrm{Sp}(\operatorname{tr})$ \\
\hline S1799 & 5 & 1500 & 3 & $\mathrm{Cpx}+\mathrm{Grt}+\mathrm{M}$ & $\mathrm{Ol}+\mathrm{Opx}+\mathrm{Cpx}+\mathrm{Grt}+\mathrm{Sp}(\operatorname{tr})$ \\
\hline S1788 & 5 & 1550 & 3 & M & $\mathrm{Ol}+\mathrm{Cpx}+\mathrm{Opx}+\mathrm{Grt}+\mathrm{Sp}(\mathrm{tr})$ \\
\hline S1790 & 5 & 1650 & 3 & M & Opx \\
\hline S1808 & 8 & 1300 & 3 & $\mathrm{Cpx}+\mathrm{Grt}+\mathrm{Coe}$ & $\mathrm{Ol}+\mathrm{Opx}+\mathrm{Cpx}+\mathrm{Grt}+\mathrm{Sp}(\mathrm{tr})$ \\
\hline S1812 & 8 & 1500 & 3 & $\mathrm{Cpx}+\mathrm{Grt}+\mathrm{Coe}$ & $\mathrm{Ol}+\mathrm{Opx}+\mathrm{Cpx}+\mathrm{Grt}+\mathrm{Sp}(\mathrm{tr})$ \\
\hline
\end{tabular}

"tr" represents for trace amount of minerals or melts which cannot be analyzed by EPMA. 


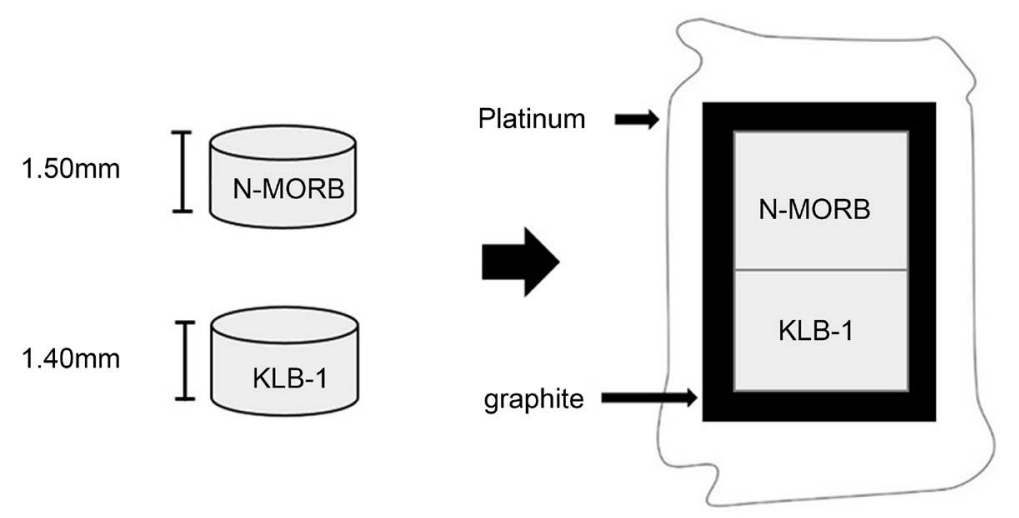

Figure 2. Capsule assemblage used for layered experiments.

Experiments performed at 2.9 GPa were conducted using a Boyd-England-type piston-cylinder apparatus, and were based on a half-inch calibration of talc/Pyrexglass/graphite assembly [4] with W-5\%Re, W-26\%Re thermocouples (a cross section of this configuration is shown in Figure 3(a)). In those runs, the twolithology starting material was kept at $1200^{\circ} \mathrm{C}$ and $2.9 \mathrm{GPa}$ for 20 hours before the final experiment in order to convert low-pressure mineral assemblages to subsolidus high-pressure eclogite and garnet peridotite assemblages. Conversely, $5 \mathrm{GPa}$ and $8 \mathrm{GPa}$ experiments were performed using a 1000-ton end-loaded Kawai-type multi-anvil apparatus [19]. We used $18 \mathrm{~mm}$ edge-length $\mathrm{MgO}$ octahedra as the pressure medium, and FUJILLOY (TN-05) tungsten carbide anvils with truncation edge lengths of $11 \mathrm{~mm} . \mathrm{LaCrO}_{3}$ was employed as the heater, and $\mathrm{W}-5 \% \mathrm{Re}$ and $\mathrm{W}-26 \%$ Re were used as thermocouples (Figure 3(a)). The pressure calibration of Aoki and Takahashi [20] was used.

\subsection{Analytical Methods}

Run products were collected, mounted in epoxy, polished, and carbon-coated for electron microprobe analysis (EPMA). Textures and chemical compositions were analysed with a JEOL-JXA8530F electron microprobe housed at the ELSI, Tokyo Institute of Technology. The acceleration voltage, beam current, and peak measurement time were $15.0 \mathrm{kV}, 1 \times 10^{-8} \mathrm{~A}$ and $20 \mathrm{~s}$, respectively. $\mathrm{Na}$ was analysed for $10 \mathrm{~s}$ in order to avoid count loss. Mineral grains were analysed using a focused electron beam, while a defocused beam $(\sim 5-30 \mu \mathrm{m})$ was used for silicate melt.

\section{Results and Discussions}

\subsection{Phase Assemblages and Textures}

All run conditions and the phases produced in each lithology are listed in Table 2. The eclogite layer contained clinopyroxene (Cpx), plagioclase (Pl), garnet (Grt), and coesite (Coe) at subsolidus temperatures, and Cpx, Grt, and silicate melt at suprasolidus temperatures (only a low degree of partial melting was observed). Basaltic melts coexisted with Cpx and Grt in isolated subsolidus peridotite layers. The peridotite failed to melt in most of our experiments, where it consisted 
(a) Cross Section of Piston Cylinder Run Products
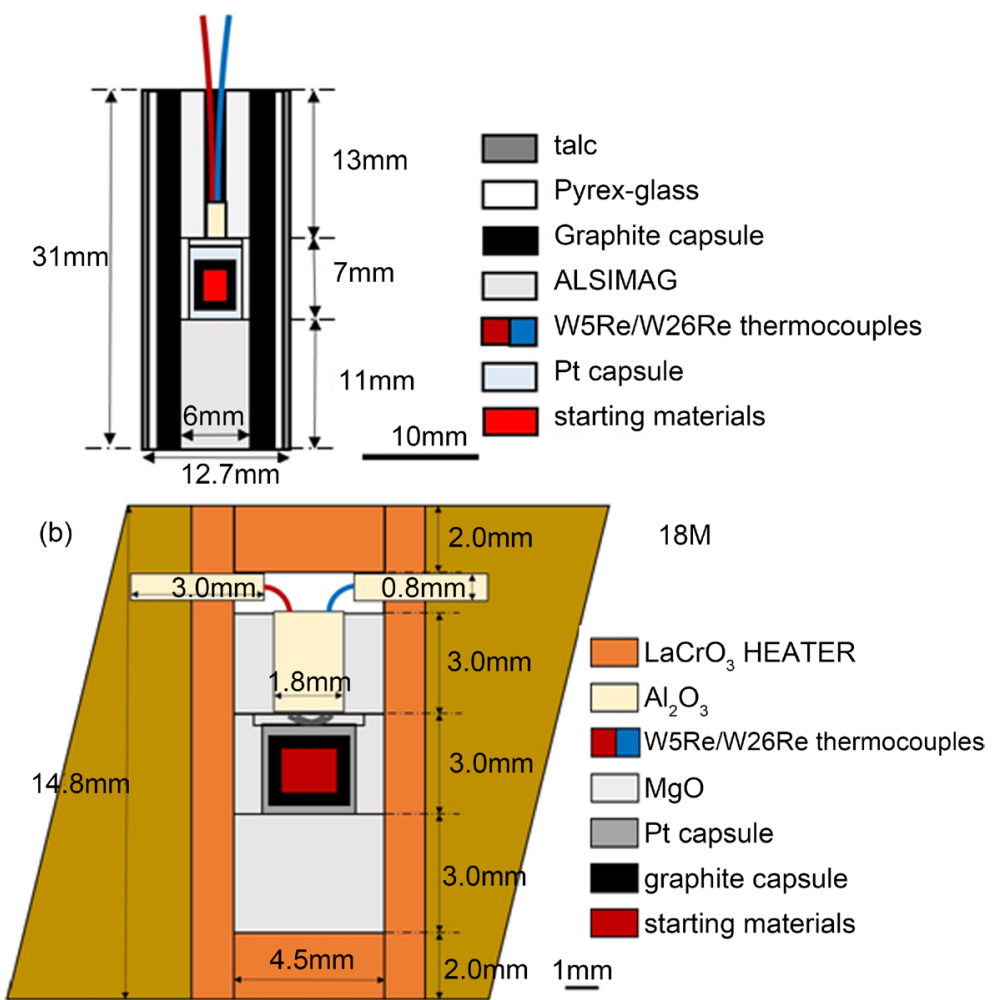

(c) Work flow diagram

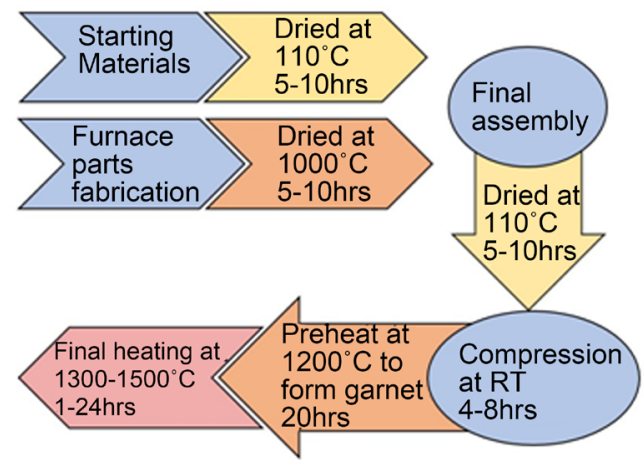

Figure 3. (a) Cross section for the $2.9 \mathrm{GPa}$ piston cylinder experiments with talc/Pyrexglass/graphite assembly. (b) Cross section for 5 and $8 \mathrm{GPa}$ multi-anvil experiments with $\mathrm{MgO} / \mathrm{LaCrO}_{3} / \mathrm{MgO}$ assembly. (c) Work flow diagram of the experimental procedures.

of olivine (Oliv), orthopyroxene (Opx), Cpx, and Grt. An additional Al-rich phase (Cr-rich spinel) was present in 2.9-GPa run products, though this transformed to garnet at higher pressures. Significant melting took place in runs conducted at temperatures above the peridotite solidus-particularly near the basalt melt layer-with Oliv and Opx present as restitic phases in the suprasolidus regime. Melt pockets spread along matrix Opx-Oliv grain boundaries, while Cpx, Grt, and spinel were consumed.

Cpx and Grt produced at subsolidus basalt temperatures were mostly subhedral to euhedral (Figure 4(a), Figure 4(b)), although traces of melt occurred along 
their grain boundaries at temperatures slightly above the dry eclogite solidus (Figure 4(d), Figure 4(e)). In the peridotite layer, Opx was mostly euhedral (Figure 4(c), Figure 4(f)), olivine occurred along Opx-Cpx-garnet grain boundaries (i.e., within the peridotite matrix), and garnet had a poikilitic texture.

\subsection{Reaction Band of $0 \mathrm{px}$}

Since partial melt formed in eclogite at pressure-temperature conditions below the peridotite solidus would not have been in chemical equilibrium with Oliv, thin Opx reaction bands formed between both rock types via chemical reaction between silica-rich partial melt of eclogite and Oliv in the peridotite matrix. At subsolidus peridotite conditions, the thickness of the Opx reaction band positively correlated with the run temperature and durations. As partially melted eclogite layers were separated from peridotite by thin Opx bands, chemical reactions between each basalt and peridotite layer were thus hindered and could only proceed via solid-state diffusion. At higher temperatures, substantial melting occurred both in basalt and peridotite layers, and fully molten basalt melts merged with molten pockets in peridotite. Extensive chemical mass transport was able to take place through chemical diffusion and liquid percolation under these conditions, and so basalt melting consistently allowed chemical equilibrium to be established with ambient peridotite, even in short runs.

\subsection{Phase and Melt Compositions}

Representative chemical compositions of individual solid and melt phases are listed in Table 3. Melts produced at all experimental conditions exhibited compositions that plotted in the basalt to basaltic andesite domains on a Harker diagram (Figure 5). Black crosses represent data from Mallik and Dasgupta [17]. Basaltic andesite melts dominated peridotite subsolidus runs, while relatively

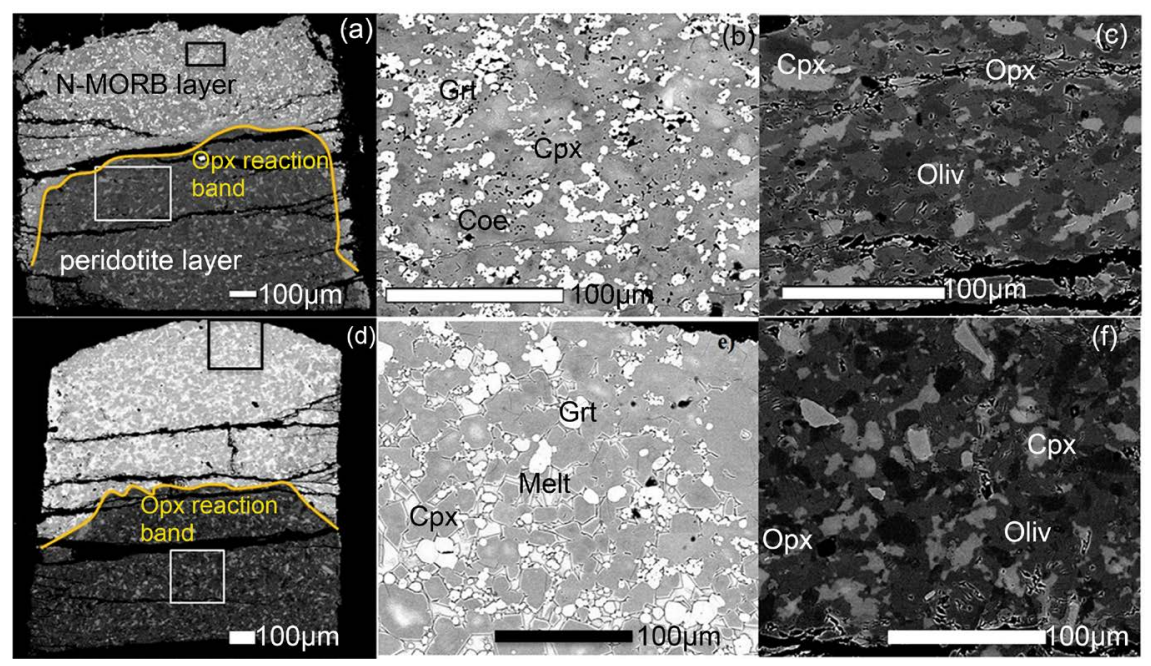

Figure 4. Backscattered electron images of run products. a) P951, $2.9 \mathrm{GPa}, 1300^{\circ} \mathrm{C}, 24 \mathrm{~h}$; b) P951 basalt layer; c) P951 peridotite layer; d) P931, $2.9 \mathrm{GPa}, 1460^{\circ} \mathrm{C}, 24 \mathrm{~h}$; e) P931 basalt layer; f) P931 peridotite layer. 
Table 3. Representative chemical compositions of individual phase in weight percent.

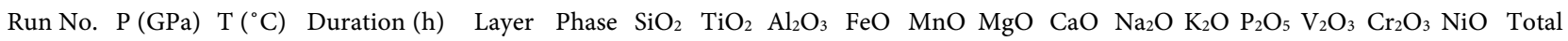
$\begin{array}{llll}\mathrm{P} 948 & 2.9 & 1300 & 3\end{array}$ $\begin{array}{lllllllllllllll}\text { MORB Cpx1 } & 52.46 & 0.80 & 5.64 & 10.44 & 0.19 & 15.99 & 10.91 & 2.12 & 0.00 & 0.09 & 0.00 & 0.00 & 0.03 & 98.68\end{array}$ $\begin{array}{lllllllllllllll}\text { Opx } & 53.17 & 0.45 & 2.96 & 12.14 & 0.18 & 26.58 & 2.61 & 0.41 & 0.00 & 0.00 & 0.00 & 0.00 & 0.12 & 98.61\end{array}$ $\begin{array}{lllllllllllllll}\text { Grt } & 38.00 & 0.88 & 20.50 & 22.20 & 0.41 & 7.39 & 8.92 & 0.13 & 0.01 & 0.16 & 0.00 & 0.00 & 0.01 & 98.60\end{array}$ Pl $\quad \begin{array}{llllllllllllll}51.55 & 0.09 & 29.73 & 0.60 & 0.00 & 0.10 & 12.51 & 3.92 & 0.62 & 0.09 & 0.00 & 0.00 & 0.02 & 99.24\end{array}$ $\begin{array}{lllllllllllllll}\text { Rt } & 5.75 & 79.85 & 3.46 & 5.24 & 0.06 & 1.54 & 1.63 & 0.05 & 0.01 & 0.00 & 7.91 & 0.00 & 0.01 & 105.51\end{array}$ $\begin{array}{llllllllllllllll}\text { KLB-1 } & \text { Ol } & 39.98 & 0.01 & 0.04 & 10.22 & 0.14 & 48.32 & 0.13 & 0.02 & 0.00 & 0.02 & 0.00 & 0.00 & 0.37 & 99.25\end{array}$ $\begin{array}{lllllllllllllll}\text { Opx } & 53.70 & 0.12 & 4.95 & 6.52 & 0.14 & 32.36 & 0.86 & 0.12 & 0.00 & 0.01 & 0.00 & 0.00 & 0.10 & 98.88\end{array}$ $\begin{array}{lllllllllllllll}\text { Cpx } & 51.02 & 0.58 & 7.09 & 3.31 & 0.09 & 15.16 & 19.47 & 1.66 & 0.00 & 0.00 & 0.00 & 0.00 & 0.05 & 98.42\end{array}$ $\begin{array}{lllllllllllllll}\text { Sp } & 0.12 & 0.14 & 56.69 & 10.22 & 0.12 & 20.53 & 0.06 & 0.00 & 0.00 & 0.00 & 0.00 & 4.52 & 0.32 & 92.72\end{array}$

$\begin{array}{llll}\text { P951 } & 2.9 & 1300 & 24\end{array}$

$\begin{array}{llll}\mathrm{P} 928 & 2.9 & 1460 & 1\end{array}$

$\begin{array}{llll}\text { P931 } & 2.9 & 1460 & 24\end{array}$

P929 $\quad 2.9 \quad 1500 \quad 1$

$\begin{array}{llll}\mathrm{P} 935 & 2.9 & 1500 & 24\end{array}$ $\begin{array}{llllllllllllllll}\text { MORB } & \text { Cpx } & 50.56 & 1.64 & 13.97 & 6.99 & 0.09 & 7.65 & 13.93 & 3.83 & 0.01 & 0.07 & 0.00 & 0.00 & 0.04 & 98.76\end{array}$ $\begin{array}{lllllllllllllll}\text { Grt } & 39.16 & 0.86 & 21.45 & 18.63 & 0.40 & 9.01 & 9.15 & 0.18 & 0.01 & 0.21 & 0.00 & 0.00 & 0.01 & 99.09\end{array}$ $\begin{array}{lllllllllllllll}\text { Coe } & 93.75 & 0.07 & 1.49 & 0.89 & 0.01 & 0.45 & 0.53 & 0.02 & 0.00 & 0.00 & 0.00 & 0.00 & 0.00 & 97.20\end{array}$ $\begin{array}{lllllllllllllll}\text { MORB Cpx1 } & 50.60 & 0.95 & 13.18 & 8.00 & 0.16 & 8.96 & 13.47 & 3.34 & 0.02 & 0.01 & 0.00 & 0.00 & 0.03 & 98.73\end{array}$

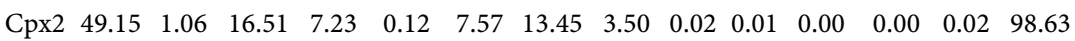
$\begin{array}{lllllllllllllll}\text { Сpx3 } & 52.51 & 0.78 & 7.26 & 9.75 & 0.18 & 14.81 & 10.60 & 2.32 & 0.00 & 0.03 & 0.00 & 0.00 & 0.04 & 98.28\end{array}$ $\begin{array}{lllllllllllllll}\text { Grt } & 38.75 & 0.92 & 21.17 & 18.44 & 0.42 & 9.75 & 8.32 & 0.18 & 0.01 & 0.09 & 0.00 & 0.00 & 0.02 & 98.07\end{array}$ $\begin{array}{lllllllllllllll}\text { M } & 54.39 & 3.63 & 14.70 & 9.30 & 0.11 & 2.21 & 7.39 & 3.23 & 1.12 & 0.44 & 0.00 & 0.00 & 0.01 & 96.53\end{array}$ $\begin{array}{llllllllllllllll}\text { KLB-1 } & \text { Ol } & 40.31 & 0.03 & 0.11 & 10.52 & 0.14 & 47.40 & 0.21 & 0.03 & 0.00 & 0.02 & 0.00 & 0.00 & 0.36 & 99.13\end{array}$ $\begin{array}{lllllllllllllll}\text { Opx } & 54.41 & 0.13 & 4.94 & 6.59 & 0.15 & 31.92 & 0.86 & 0.12 & 0.01 & 0.04 & 0.00 & 0.00 & 0.11 & 99.28\end{array}$ $\begin{array}{lllllllllllllll}\text { Cpx } & 51.78 & 0.54 & 7.08 & 3.44 & 0.08 & 15.21 & 19.06 & 1.49 & 0.00 & 0.01 & 0.00 & 0.00 & 0.06 & 98.75\end{array}$ $\begin{array}{lllllllllllllll}\text { Sp } & 0.069 & 0.12 & 56.76 & 9.86 & 0.12 & 20.83 & 0.03 & 0.00 & 0.01 & 0.00 & 0.00 & 5.22 & 0.35 & 93.36\end{array}$

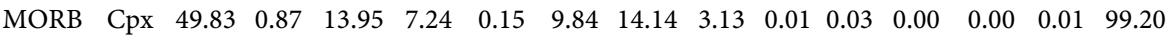
$\begin{array}{lllllllllllllll}\text { Grt } & 39.68 & 0.76 & 22.08 & 15.95 & 0.41 & 11.94 & 8.24 & 0.11 & 0.00 & 0.04 & 0.00 & 0.00 & 0.01 & 99.23\end{array}$ $\begin{array}{lllllllllllllll}\text { M } & 52.66 & 2.83 & 15.12 & 10.40 & 0.14 & 4.19 & 8.66 & 3.22 & 0.68 & 0.27 & 0.00 & 0.00 & 0.00 & 98.18\end{array}$ $\begin{array}{llllllllllllllll}\text { KLB-1 } & \text { Ol } & 40.55 & 0.01 & 0.12 & 10.44 & 0.12 & 48.15 & 0.25 & 0.03 & 0.00 & 0.02 & 0.00 & 0.00 & 0.36 & 100.06\end{array}$ $\begin{array}{lllllllllllllll}\text { Opx } & 54.72 & 0.15 & 4.99 & 6.55 & 0.13 & 32.03 & 1.06 & 0.16 & 0.01 & 0.05 & 0.00 & 0.00 & 0.12 & 99.98\end{array}$ $\begin{array}{lllllllllllllll}\text { Sp } & 0.37 & 0.34 & 57.31 & 9.39 & 0.12 & 21.31 & 0.04 & 0.02 & 0.00 & 0.00 & 0.00 & 4.98 & 0.32 & 94.18\end{array}$ $\begin{array}{llllllllllllllll}\text { MORB } & \text { Cpx } & 49.50 & 0.71 & 13.37 & 6.70 & 0.16 & 10.32 & 14.61 & 2.95 & 0.01 & 0.02 & 0.00 & 0.00 & 0.03 & 98.38\end{array}$ $\begin{array}{lllllllllllllll}\text { Grt } & 39.22 & 0.79 & 21.39 & 16.17 & 0.39 & 11.92 & 7.98 & 0.16 & 0.01 & 0.07 & 0.00 & 0.00 & 0.04 & 98.15\end{array}$ $\begin{array}{lllllllllllllll}M & 51.21 & 2.62 & 15.34 & 11.38 & 0.17 & 3.72 & 8.45 & 2.93 & 0.62 & 0.26 & 0.00 & 0.00 & 0.01 & 96.70\end{array}$ $\begin{array}{llllllllllllllll}\text { KLB-1 } & \text { Ol } & 39.88 & 0.01 & 0.15 & 10.73 & 0.14 & 47.56 & 0.29 & 0.03 & 0.00 & 0.06 & 0.00 & 0.00 & 0.39 & 99.22\end{array}$ $\begin{array}{lllllllllllllll}\text { Opx } & 53.89 & 0.15 & 4.95 & 6.71 & 0.13 & 32.10 & 0.88 & 0.13 & 0.01 & 0.02 & 0.00 & 0.00 & 0.11 & 99.07\end{array}$ $\begin{array}{lllllllllllllll}\text { Cpx } & 50.95 & 0.61 & 7.13 & 3.28 & 0.08 & 15.21 & 19.12 & 1.63 & 0.01 & 0.04 & 0.00 & 0.00 & 0.06 & 98.12\end{array}$ $\begin{array}{lllllllllllllll}\text { Sp } & 0.07 & 0.12 & 56.76 & 9.86 & 0.12 & 20.83 & 0.03 & 0.00 & 0.01 & 0.00 & 0.00 & 5.22 & 0.35 & 93.36\end{array}$

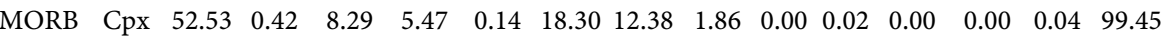
$\begin{array}{lllllllllllllll}\text { Grt } & 41.16 & 0.56 & 22.39 & 10.21 & 0.31 & 17.85 & 6.57 & 0.04 & 0.01 & 0.03 & 0.00 & 0.00 & 0.01 & 99.14\end{array}$ $\begin{array}{lllllllllllllll}\text { M } & 47.49 & 2.01 & 12.87 & 8.98 & 0.16 & 12.21 & 8.68 & 2.78 & 0.42 & 0.24 & 0.00 & 0.00 & 0.01 & 95.83\end{array}$ 


\section{Continued}

\begin{tabular}{|c|c|c|c|c|c|c|c|c|c|c|c|c|c|c|c|c|c|c|c|}
\hline & & & & KLB-1 & $\mathrm{Ol}$ & 40.58 & 0.02 & 0.15 & 10.32 & 0.14 & 48.17 & 0.30 & 0.03 & 0.00 & 0.01 & 0.00 & 0.00 & 0.34 & 100.07 \\
\hline & & & & & Opx & 54.64 & 0.15 & 4.99 & 6.38 & 0.14 & 31.97 & 1.08 & 0.17 & 0.00 & 0.03 & 0.00 & 0.00 & 0.13 & 99.69 \\
\hline \multirow[t]{7}{*}{ P932 } & 2.9 & 1540 & 1 & MORB & Cpx & 50.35 & 0.70 & 11.99 & 6.27 & 0.16 & 13.43 & 14.64 & 2.10 & 0.01 & 0.02 & 0.00 & 0.00 & 0.01 & 99.67 \\
\hline & & & & & $S p$ & 0.12 & 0.42 & 57.43 & 10.09 & 0.08 & 20.73 & 0.09 & 0.02 & 0.01 & 0.00 & 0.00 & 5.59 & 0.38 & 94.95 \\
\hline & & & & & $\mathrm{M}$ & 48.76 & 1.60 & 13.93 & 9.79 & 0.18 & 9.45 & 10.95 & 2.59 & 0.24 & 0.11 & 0.00 & 0.00 & 0.01 & 97.62 \\
\hline & & & & KLB-1 & $\mathrm{Ol}$ & 40.57 & 0.01 & 0.16 & 10.33 & 0.14 & 48.13 & 0.30 & 0.04 & 0.01 & 0.00 & 0.00 & 0.00 & 0.35 & 100.04 \\
\hline & & & & & Opx & 54.73 & 0.14 & 4.94 & 6.57 & 0.15 & 32.30 & 0.92 & 0.11 & 0.01 & 0.02 & 0.00 & 0.00 & 0.09 & 99.96 \\
\hline & & & & & Cpx & 52.73 & 0.28 & 6.86 & 5.31 & 0.12 & 23.02 & 9.88 & 0.86 & 0.01 & 0.02 & 0.00 & 0.00 & 0.11 & 99.20 \\
\hline & & & & & Grt & 42.34 & 0.66 & 21.75 & 6.84 & 0.20 & 21.25 & 5.46 & 0.08 & 0.04 & 0.12 & 0.00 & 0.00 & 0.03 & 98.76 \\
\hline \multirow[t]{2}{*}{ P936 } & 2.9 & 1540 & 24 & MORB & M & 48.14 & 1.09 & 11.00 & 6.44 & 0.17 & 19.90 & 8.64 & 1.81 & 0.18 & 0.06 & 0.00 & 0.00 & 0.01 & 97.43 \\
\hline & & & & KLB-1 & $\mathrm{Ol}$ & 41.59 & 0.01 & 0.17 & 5.95 & 0.11 & 52.60 & 0.23 & 0.02 & 0.01 & 0.04 & 0.00 & 0.00 & 0.01 & 100.74 \\
\hline \multirow[t]{7}{*}{ S1813 } & 5 & 1300 & 3 & MORB & Cpx & 51.61 & 1.25 & 15.46 & 5.93 & 0.08 & 6.87 & 12.69 & 4.53 & 0.16 & 0.09 & 0.00 & 0.00 & 0.03 & 98.70 \\
\hline & & & & & Grt & 39.85 & 1.10 & 20.99 & 18.03 & 0.37 & 8.84 & 10.08 & 0.31 & 0.08 & 0.22 & 0.00 & 0.00 & 0.02 & 99.89 \\
\hline & & & & & $\mathrm{Coe}$ & 97.54 & 0.11 & 0.52 & 0.48 & 0.02 & 0.11 & 0.24 & 0.01 & 0.01 & 0.00 & 0.00 & 0.00 & 0.00 & 99.05 \\
\hline & & & & KLB-1 & $\mathrm{Ol}$ & 40.11 & 0.02 & 0.04 & 10.23 & 0.14 & 48.92 & 0.11 & 0.01 & 0.00 & 0.04 & 0.00 & 0.00 & 0.35 & 99.97 \\
\hline & & & & & Opx & 54.04 & 0.13 & 4.88 & 6.52 & 0.15 & 32.58 & 0.88 & 0.11 & 0.01 & 0.05 & 0.00 & 0.00 & 0.11 & 99.45 \\
\hline & & & & & Cpx & 51.44 & 0.58 & 6.80 & 3.36 & 0.08 & 15.65 & 19.22 & 1.66 & 0.00 & 0.05 & 0.00 & 0.00 & 0.07 & 98.93 \\
\hline & & & & & $S p$ & 0.14 & 0.15 & 57.44 & 10.50 & 0.11 & 20.92 & 0.05 & 0.01 & 0.00 & 0.04 & 0.00 & 4.64 & 0.40 & 94.37 \\
\hline \multirow[t]{8}{*}{ S1797 } & 5 & 1450 & 3 & MORB & Cpx & 52.68 & 1.01 & 13.42 & 6.75 & 0.11 & 9.50 & 13.16 & 3.91 & 0.04 & 0.01 & 0.15 & 0.05 & 0.02 & 100.82 \\
\hline & & & & & Grt & 41.34 & 0.80 & 22.39 & 15.04 & 0.36 & 12.53 & 8.55 & 0.11 & 0.00 & 0.07 & 0.10 & 0.09 & 0.01 & 101.39 \\
\hline & & & & & $\mathrm{M}$ & 51.78 & 2.38 & 14.59 & 9.96 & 0.15 & 5.91 & 8.95 & 3.37 & 0.60 & 0.16 & 0.27 & 0.01 & 0.02 & 98.14 \\
\hline & & & & KLB-1 & $\mathrm{Ol}$ & 41.35 & 0.02 & 0.08 & 11.72 & 0.12 & 48.64 & 0.20 & 0.05 & 0.01 & 0.01 & 0.01 & 0.07 & 0.38 & 102.66 \\
\hline & & & & & Opx & 55.88 & 0.12 & 4.79 & 6.68 & 0.13 & 33.12 & 0.81 & 0.16 & 0.00 & 0.01 & 0.02 & 0.27 & 0.13 & 102.13 \\
\hline & & & & & Cpx & 53.35 & 0.56 & 7.66 & 4.31 & 0.09 & 16.27 & 15.65 & 2.26 & 0.01 & 0.00 & 0.07 & 0.38 & 0.07 & 100.67 \\
\hline & & & & & Grt & 42.41 & 0.73 & 22.88 & 10.86 & 0.27 & 16.95 & 7.23 & 0.12 & 0.00 & 0.00 & 0.11 & 0.02 & 0.01 & 101.58 \\
\hline & & & & & $\mathrm{Sp}$ & 0.15 & 0.53 & 58.35 & 9.54 & 0.09 & 22.06 & 0.07 & 0.00 & 0.00 & 0.00 & 0.11 & 7.88 & 0.41 & 99.18 \\
\hline \multirow[t]{7}{*}{ S1802 } & 5 & 1450 & 20 & MORB & Cpx & 52.90 & 1.09 & 14.07 & 7.09 & 0.11 & 8.04 & 11.43 & 4.17 & 0.01 & 0.02 & 0.15 & 0.03 & 0.02 & 99.13 \\
\hline & & & & & Grt & 40.58 & 0.98 & 22.05 & 17.68 & 0.39 & 9.77 & 8.12 & 0.19 & 0.01 & 0.02 & 0.13 & 0.01 & 0.03 & 99.94 \\
\hline & & & & & $\mathrm{M}$ & 54.84 & 3.57 & 12.93 & 8.62 & 0.10 & 3.29 & 6.55 & 2.89 & 1.13 & 0.09 & 0.39 & 0.01 & 0.01 & 94.43 \\
\hline & & & & KLB-1 & $\mathrm{Ol}$ & 41.12 & 0.03 & 0.08 & 10.55 & 0.10 & 48.90 & 0.17 & 0.02 & 0.01 & 0.00 & 0.00 & 0.05 & 0.41 & 101.45 \\
\hline & & & & & Opx & 55.07 & 0.18 & 4.32 & 6.57 & 0.11 & 32.40 & 1.01 & 0.19 & 0.00 & 0.00 & 0.04 & 0.30 & 0.13 & 100.32 \\
\hline & & & & & Cpx & 52.48 & 0.52 & 6.56 & 3.58 & 0.08 & 16.19 & 16.87 & 1.56 & 0.01 & 0.01 & 0.09 & 0.61 & 0.08 & 98.64 \\
\hline & & & & & Grt & 41.97 & 0.61 & 21.24 & 7.10 & 0.20 & 21.18 & 4.54 & 0.01 & 0.01 & 0.00 & 0.09 & 2.27 & 0.02 & 99.23 \\
\hline \multirow[t]{5}{*}{ S1799 } & 5 & 1500 & 3 & MORB & $\mathrm{Cpx}$ & 51.05 & 0.88 & 15.09 & 6.57 & 0.13 & 8.18 & 13.53 & 3.97 & 0.01 & 0.00 & 0.00 & 0.00 & 0.04 & 99.44 \\
\hline & & & & & Grt & 39.26 & 0.91 & 21.77 & 16.74 & 0.39 & 10.33 & 9.53 & 0.15 & 0.01 & 0.06 & 0.00 & 0.00 & 0.01 & 99.16 \\
\hline & & & & & $\mathrm{M}$ & 56.00 & 4.52 & 13.05 & 9.44 & 0.10 & 2.22 & 7.23 & 2.70 & 1.40 & 0.41 & 0.42 & 0.01 & 0.01 & 97.49 \\
\hline & & & & KLB-1 & $\mathrm{Ol}$ & 41.32 & 0.03 & 0.09 & 10.33 & 0.12 & 49.17 & 0.21 & 0.04 & 0.01 & 0.01 & 0.00 & 0.06 & 0.37 & 101.74 \\
\hline & & & & & Opx & 55.84 & 0.20 & 4.10 & 6.86 & 0.13 & 32.23 & 1.34 & 0.23 & 0.01 & 0.01 & 0.04 & 0.25 & 0.13 & 101.36 \\
\hline
\end{tabular}




\section{Continued}

\begin{tabular}{|c|c|c|c|c|c|c|c|c|c|c|c|c|c|c|c|c|c|c|c|}
\hline & & & & & Cpx & 53.20 & 0.46 & 5.95 & 3.73 & 0.11 & 17.76 & 17.16 & 1.43 & 0.01 & 0.02 & 0.08 & 0.58 & 0.07 & 100.54 \\
\hline & & & & & Grt & 44.76 & 0.39 & 21.19 & 7.15 & 0.25 & 21.51 & 5.70 & 0.06 & 0.01 & 0.00 & 0.05 & 1.23 & 0.03 & 102.31 \\
\hline & & & & & $\mathrm{Sp}$ & 4.78 & 0.48 & 50.17 & 10.35 & 0.12 & 22.07 & 0.48 & 0.00 & 0.01 & 0.02 & 0.16 & 11.09 & 0.40 & 100.13 \\
\hline \multirow[t]{4}{*}{ S1788 } & 5 & 1550 & 3 & MORB & M & 49.79 & 1.73 & 14.00 & 9.64 & 0.18 & 9.64 & 9.85 & 2.85 & 0.32 & 0.17 & 0.19 & 0.04 & 0.01 & 98.41 \\
\hline & & & & KLB-1 & $\mathrm{Ol}$ & 40.44 & 0.03 & 0.14 & 11.00 & 0.12 & 49.74 & 0.28 & 0.06 & 0.01 & 0.05 & 0.00 & 0.07 & 0.31 & 102.24 \\
\hline & & & & & Opx & 55.21 & 0.11 & 4.86 & 6.54 & 0.15 & 33.31 & 0.87 & 0.16 & 0.01 & 0.00 & 0.05 & 0.27 & 0.10 & 101.62 \\
\hline & & & & & Cpx & 54.33 & 0.31 & 4.68 & 5.61 & 0.15 & 25.00 & 9.49 & 0.84 & 0.01 & 0.01 & 0.07 & 0.46 & 0.06 & 101.02 \\
\hline \multirow[t]{2}{*}{ S1790 } & 5 & 1650 & 3 & MORB & M & 48.83 & 0.88 & 9.54 & 7.22 & 0.14 & 23.03 & 7.04 & 1.70 & 0.15 & 0.05 & 0.10 & 0.18 & 0.02 & 98.88 \\
\hline & & & & KLB-1 & Opx & 57.43 & 0.09 & 3.86 & 4.03 & 0.07 & 34.45 & 1.38 & 0.24 & 0.00 & 0.01 & 0.01 & 0.28 & 0.02 & 101.87 \\
\hline \multirow[t]{7}{*}{ S1808 } & 8 & 1300 & 3 & MORB & Cpx & 54.28 & 0.75 & 11.10 & 5.14 & 0.06 & 8.73 & 12.77 & 5.31 & 0.27 & 0.01 & 0.00 & 0.00 & 0.01 & 98.42 \\
\hline & & & & & Grt & 40.92 & 1.09 & 20.05 & 16.30 & 0.31 & 9.90 & 9.78 & 0.74 & 0.07 & 0.20 & 0.00 & 0.00 & 0.01 & 99.38 \\
\hline & & & & & Coe & 96.14 & 0.06 & 0.65 & 0.52 & 0.01 & 0.28 & 0.44 & 0.22 & 0.02 & 0.00 & 0.00 & 0.00 & 0.01 & 98.33 \\
\hline & & & & KLB-1 & $\mathrm{Ol}$ & 40.46 & 0.03 & 0.16 & 9.98 & 0.10 & 48.38 & 0.12 & 0.02 & 0.01 & 0.01 & 0.00 & 0.00 & 0.39 & 99.65 \\
\hline & & & & & Opx & 53.79 & 0.12 & 4.65 & 6.53 & 0.16 & 32.60 & 0.88 & 0.16 & 0.00 & 0.00 & 0.00 & 0.00 & 0.11 & 98.99 \\
\hline & & & & & $\mathrm{Cpx}$ & 51.46 & 0.49 & 6.54 & 3.36 & 0.07 & 15.86 & 19.17 & 1.59 & 0.01 & 0.01 & 0.00 & 0.00 & 0.10 & 98.66 \\
\hline & & & & & Grt & 43.74 & 0.60 & 18.70 & 8.46 & 0.29 & 21.07 & 6.40 & 0.24 & 0.01 & 0.00 & 0.00 & 0.00 & 0.00 & 99.50 \\
\hline \multirow[t]{7}{*}{ S1812 } & 8 & 1500 & 3 & MORB & Cpx & 53.89 & 1.16 & 11.53 & 5.21 & 0.07 & 8.40 & 13.02 & 4.97 & 0.22 & 0.03 & 0.00 & 0.00 & 0.02 & 98.52 \\
\hline & & & & & Grt & 38.98 & 1.52 & 20.66 & 14.91 & 0.29 & 7.73 & 13.76 & 0.47 & 0.03 & 0.10 & 0.00 & 0.00 & 0.00 & 98.45 \\
\hline & & & & & Coe & 98.78 & 0.05 & 0.12 & 0.37 & 0.01 & 0.05 & 0.11 & 0.04 & 0.00 & 0.00 & 0.00 & 0.00 & 0.03 & 99.57 \\
\hline & & & & & Opx & 54.11 & 0.18 & 4.64 & 6.43 & 0.14 & 32.33 & 0.96 & 0.21 & 0.01 & 0.03 & 0.00 & 0.00 & 0.09 & 99.12 \\
\hline & & & & & Cpx & 51.03 & 0.52 & 7.04 & 3.31 & 0.10 & 15.46 & 19.53 & 1.65 & 0.00 & 0.00 & 0.00 & 0.00 & 0.10 & 98.75 \\
\hline & & & & & Grt & 41.10 & 1.11 & 20.18 & 7.52 & 0.26 & 21.64 & 4.48 & 0.08 & 0.01 & 0.19 & 0.00 & 0.00 & 0.04 & 96.60 \\
\hline & & & & & $\mathrm{Sp}$ & 0.09 & 0.55 & 56.01 & 10.52 & 0.11 & 21.06 & 0.09 & 0.00 & 0.00 & 0.00 & 0.00 & 5.46 & 0.34 & 94.22 \\
\hline
\end{tabular}

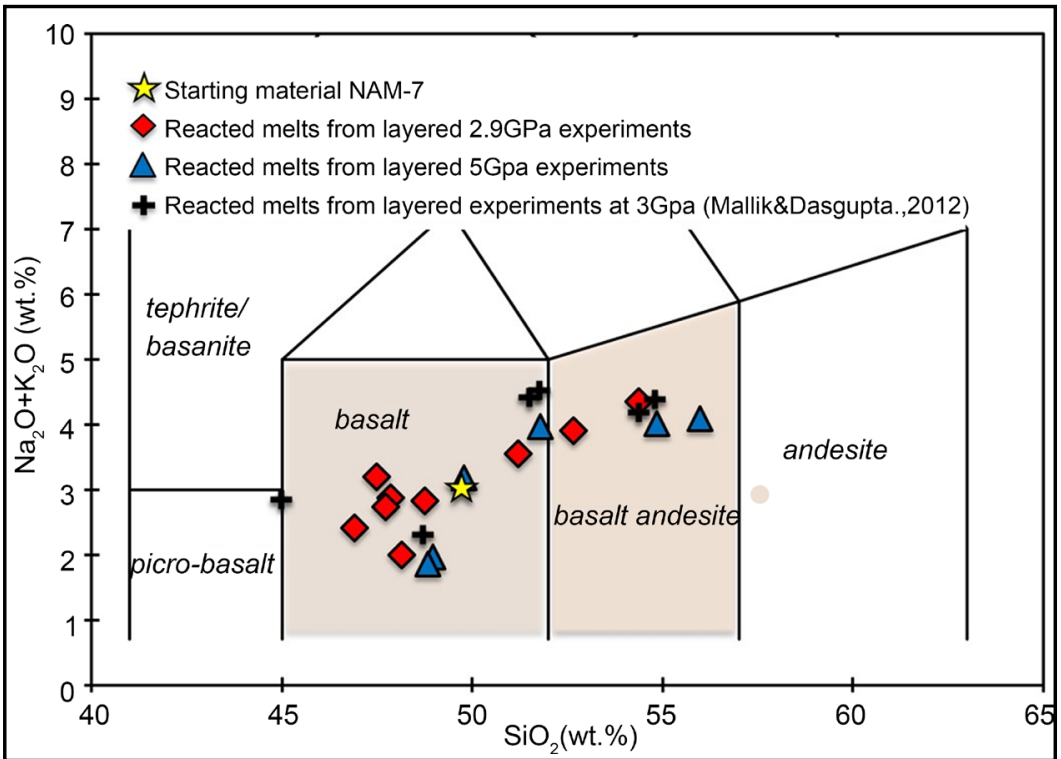

Figure 5. Total Alkali-SiO ${ }_{2}$ compositions of reacted melts. 
Si-rich tholeiitic melts dominated at temperatures near the dry peridotite solidus, the compositions of which were buffered by the aforementioned Opx reaction band along the interface of each rock type.

The relative $\mathrm{K}_{2} \mathrm{O}$ and $\mathrm{TiO}_{2}$ contents of melts formed within each starting material (yellow star in Figure 6) can be used as a proxy for the degree of melting of $\mathrm{N}$-MORB. Lower $\mathrm{K}_{2} \mathrm{O}$ and $\mathrm{TiO}_{2}$ contents mean that the volume of melt increased by melting of peridotite, which essentially lacks $\mathrm{K}_{2} \mathrm{O}$ and is very $\mathrm{TiO}_{2}-$ poor (Figure 6(c), Figure 6(d), Figure 6(f)). The presence of an Opx band caused chemical exchanges between both rock types to proceed solely by solidstate diffusion for as long as the peridotite lacked melt.

Because of the different starting materials used for layered experiments, our melt compositions (e.g., $\mathrm{Ti}, \mathrm{Na}, \mathrm{Ca}$, and $\mathrm{Fe}$ ) were systematically different from those reported by Mallik and Dasgupta [17]. In our study, all major elements except for Fe showed a close fit with a Hawaiian tholeiite composition. Partial melts of anhydrous run products contained $~ 55-57 \mathrm{wt} \% \mathrm{SiO}_{2}$ (i.e., andesite) at low temperature and $\sim 50-53 \mathrm{wt} \% \mathrm{SiO}_{2}$ (i.e., tholeiite) at high temperature; the latter melt compositions being similar to those observed in Hawaii during its shield-building stage (Figure 6(b)). Composition of melts obtained at temperatures close to the dry peridotite solidus closely matched the composition of Hawaiian tholeiite (Figure 6(a)). Notably, N-MORB had almost entirely melted at these conditions. These results and their comparison with Hawaiian tholeiite (Figure (6)) show that the chemical composition of recycled eclogite in the sub-Hawaiian plume is more enriched in $\mathrm{FeO}$ than N-MORB is.

\subsection{Implications for Hawaiian Magma Genesis}

A schematic model for melting processes in a heterogeneous Hawaiian plume is shown in Figure 7 (after Takahashi and Nakajima [7]). We have demonstrated that the entire compositional spectrum of Hawaiian tholeiites (basalt to picrite) can be formed by basalt-peridotite hybrid melting near to the dry peridotite solidus. In other words, our results indicate that the temperature at the centre of the plume head may be close to that of the dry solidus of peridotite KLB-1 $\left(1480^{\circ} \mathrm{C}\right.$ at $3 \mathrm{GPa}$, Takahashi et al. [19]), or else slightly lower. This implies that the PMT (Potential mantle Temperature) of the Hawaiian plume may be much lower than has been previously estimated (e.g., $1558^{\circ} \mathrm{C}$, Watson and McKenzie, [3]).

The size of the entrained eclogite component plays an essential role in the melting processes that occur within a heterogeneous plume. If the eclogite components were small (such as mafic dyke or mafic bands, which are common in peridotite massifs), partially molten eclogite layers could achieve equilibrium with the surrounding peridotite over geological timescales relevant to plume melting (i.e., $10^{4}-10^{6} \mathrm{yr}$ ). Conversely, if eclogite blocks were large (e.g., km-scale or larger), melt formed in the eclogite blocks would be isolated from surrounding peridotite due to the formation of Opx bands at their interfaces. The timescale of melt segregation from the partially molten eclogite may be faster 

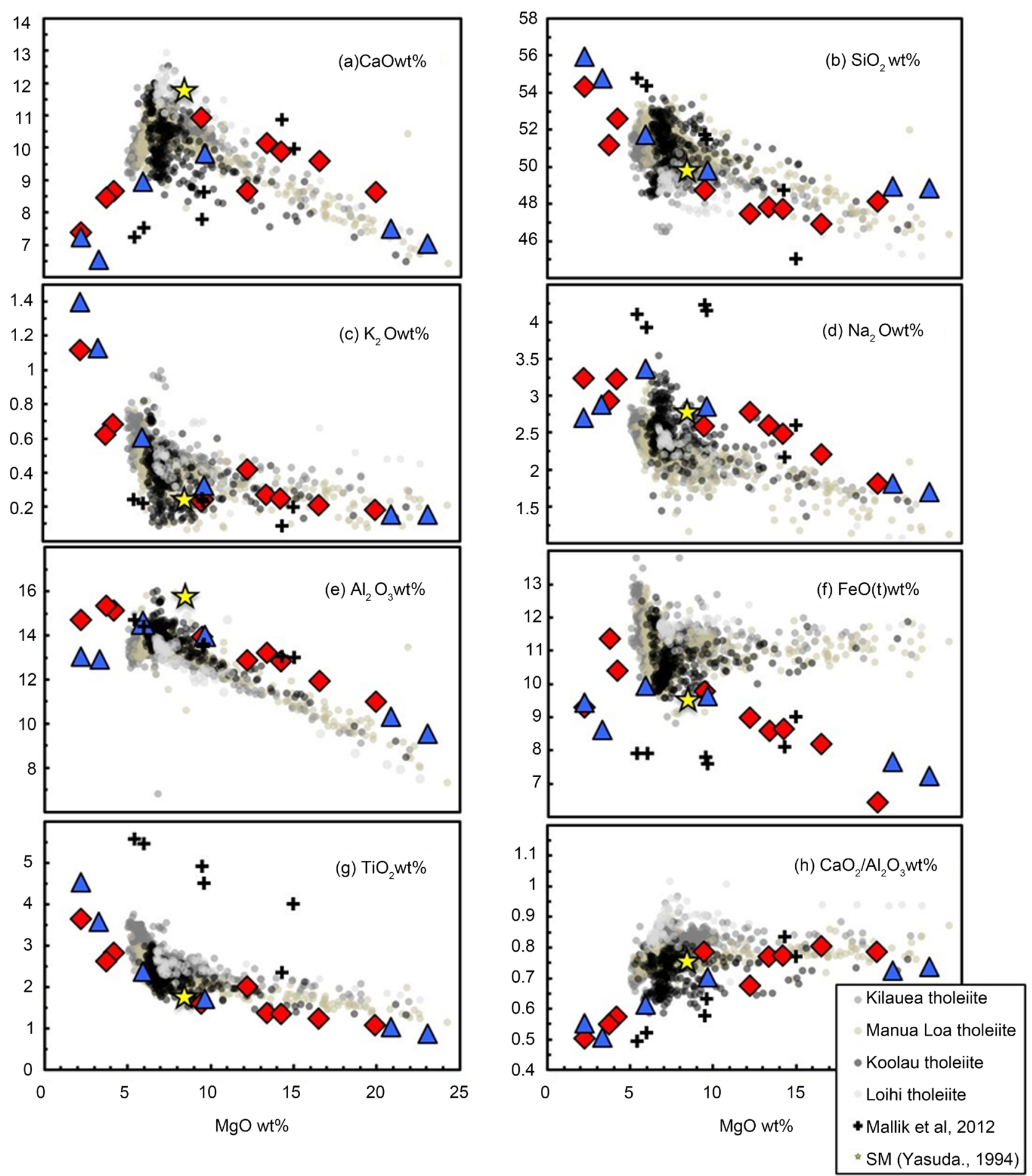

Figure 6. Major elements composition of reacted melts from layered experiments. Yellow star indicates the original composition of starting material NAM-7 [18]; red diamonds are compositions of run products conducted at 2.9 GPa, blue triangles are compositions of run products carried out at $5 \mathrm{GPa}$. Crosses represent the layered experiments data from Mallik \& Dasgupta [17]. Tholeiitic basalt components of Kilauea, Manua Loa, Koolau and Loihi are also plotted in these diagrams as black, grey dots.

than that for reaction rate across the Opx band; thus, melts derived from eclogite domains would have geochemical signatures that record entrainment and would contaminate the surrounding peridotite less extensively. 


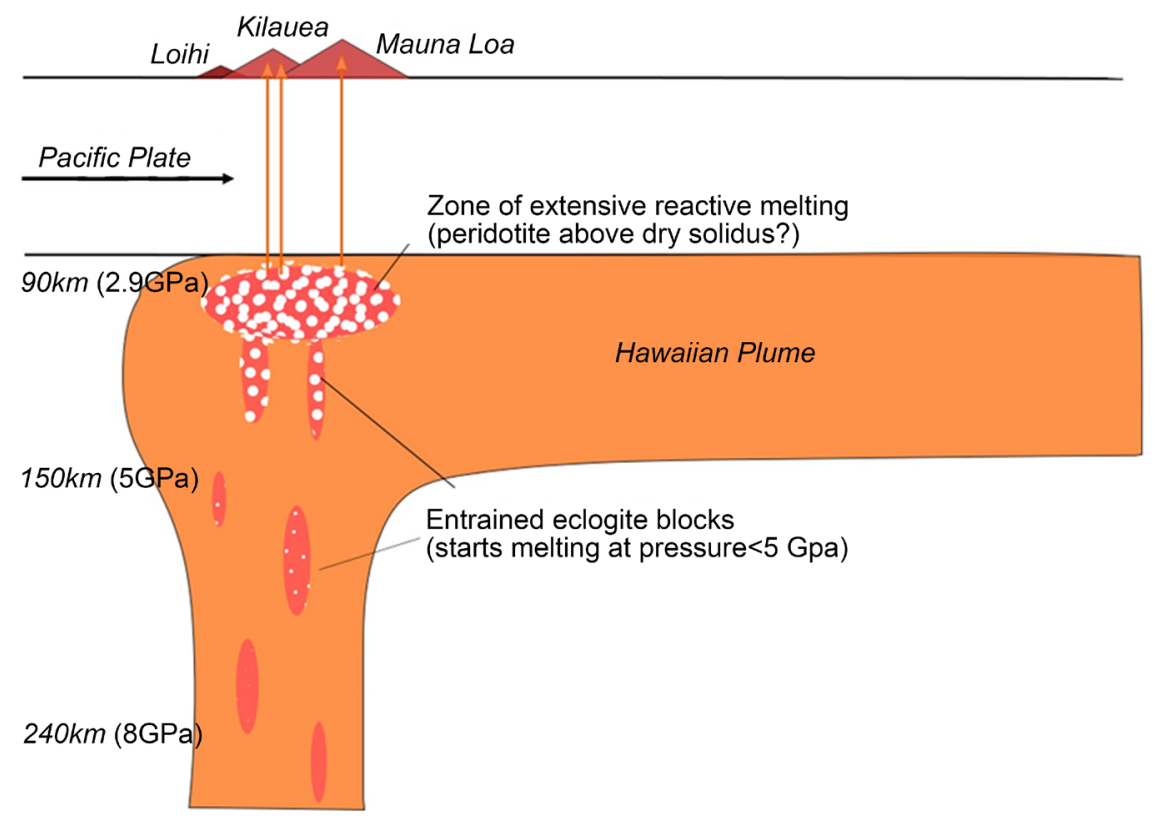

Figure 7. Schematic model of the melting process in Hawaiian plume (modified after Takahashi \& Nakajima [7]).

Takahashi and Nakajima [7] proposed that magma produced near the axis of the plume head may be a mixture of two types of melts: 1) basaltic andesite melt formed by eclogite melting, and 2) picritic melts formed by the reactive melting of eclogite and peridotite. According to their study of the Koolau volcano, an eclogite block larger than $1000 \mathrm{~km}^{3}$ supplied silica-rich magma at the last stage the volcano's formation, and remained in disequilibrium with peridotite during its lifetime. Hence layered eclogite-peridotite experiments are necessary in order to study the dynamics of the recycled oceanic crust in the ascending plume.

Tholeiite to basaltic andesite magmas could be generated in an upwelling mantle plume containing a recycled oceanic component (Figure 7) by hybridised melting of basalt and peridotite. High-silica tholeiitic OIB, such as occurs in Hawaii, may be formed under nearly dry conditions in a heterogeneous plume. The relative stability of Opx caused it to form in our experiments via chemical reaction between silicate melt and $\mathrm{SiO}_{2}$-undersaturated olivine from ambient peridotite. This Opx separated basaltic domains and peridotite domains during melting. Major-element compositions of melt derived from layered N-MORB and spinel lherzolite closely matched those of Hawaiian tholeiite. Very depleted heavy rare earth element (HREE) patterns in Hawaiian tholeiite might be attributed to percolation of partial melt in vertically elongated eclogite blobs in the centre of the plume. Enrichments in $\mathrm{Fe}, \mathrm{K}, \mathrm{Ti}$, and light rare earth elements (LREE) in the Hawaiian tholeiite compared with modern MORB may indicate that the source of the recycled components in the Hawaiian plume is more enriched in $\mathrm{Fe}$ and incompatible elements than modern N-MORB is. A likely source material here is Fe-rich tholeiite, which was common in the Archaean and early Proterozoic [21]. This conclusion is consistent with model ages of Hawaiian magma sources determined by $\mathrm{Pb}$ isotopes [21]. 


\section{Conclusions}

We have carried out high-pressure melting experiments using an N-MORB/ peridotite (KLB-1) layered starting material in order to study magma genesis processes in the heterogeneous Hawaiian plume, which has entrained ancient oceanic crust. Major conclusions from this study are as follows:

1) In an upwelling mantle plume with a recycled oceanic component, N-MORB eclogite begins to melt at depth $(5 \mathrm{GPa})$, but peridotite remains entirely solid almost up to the top of plume ( 3 GPa).

2) Extensive partial melting occurs in eclogite domains, which produces tholeiite to basaltic andesite melts. Some chemical reaction occurs at the basaltperidotite interface.

3) Excluding $\mathrm{FeO}$ contents, the major-element composition of melts formed by reactive melting of $\mathrm{N}-\mathrm{MORB} / \mathrm{KLB}-1$ at temperatures near the dry peridotite solidus are similar to Hawaiian tholeiite and picrite.

4) Relatively Fe- and LREE-enriched geochemical signatures in Hawaiian tholeiite compared with MORB suggest that the source of recycled components in the Hawaiian plume is more enriched in Fe and in incompatible elements than modern MORB.

5) The potential temperature of the Hawaiian plume could be $1450^{\circ} \mathrm{C}$ or lower.

\section{References}

[1] Sleep, N.H. (1990) Hotspots and Mantle Plumes: Some Phenomenology. Journal of Geophysical Research: Solid Earth, 95, 6715-6736.

https://doi.org/10.1029/JB095iB05p06715

[2] Clague, D.A. and Dalrymple, G.B. (1987) The Hawaiian-Emperor Volcanic Chain. Part I. Geologic Evolution. Volcanism in Hawaii, 1, 5-54.

[3] Watson, S. and McKenzie, D.P. (1991) Melt Generation by Plumes: A Study of Hawaiian Volcanism. Journal of Petrology, 32, 501-537. https://doi.org/10.1093/petrology/32.3.501

[4] Takahashi, E. (1986) Melting of a Dry Peridotite KLB-1 up to 14 GPa: Implications on the Origin of Peridotitic Upper Mantle. Journal of Geophysical Research: Solid Earth, 91, 9367-9382. https://doi.org/10.1029/JB091iB09p09367

[5] Hirose, K. and Kushiro, I. (1993) Partial Melting of Dry Peridotites at High Pressures: Determination of Compositions of Melts Segregated from Peridotite Using Aggregates of Diamond. Earth and Planetary Science Letters, 114, 477-489. https://doi.org/10.1016/0012-821X(93)90077-M

[6] Hauri, E.H. (1996) Major-Element Variability in the Hawaiian Mantle Plume. Nature, 382, 415-419. https://doi.org/10.1038/382415a0

[7] Takahashi, E. and Nakajima, K. (2002) Melting Process in the Hawaiian Plume: An Experimental Study. In: Hawaiian Volcanoes. Deep Underwater Perspectives, American Geophysical Union, Geophysical Monograph Vol. 128, 403-418. https://doi.org/10.1029/GM128p0403

[8] Ren, Z.-Y. (2004) Petrogenesis of Tholeiitic Lavas from the Submarine Hana Ridge, Haleakala Volcano, Hawaii. Journal of Petrology, 45, 2067-2099. https://doi.org/10.1093/petrology/egh076

[9] Ren, Z.-Y., Ingle, S., Takahashi, E., Hirano, N. and Hirata, T. (2005) The Chemical Structure of the Hawaiian Mantle Plume. Nature, 436, 837-840. 
https://doi.org/10.1038/nature03907

[10] Sobolev, A.V., Hofmann, A., Sobolev, S. and Nikogosian, I. (2005) An Olivine-Free Mantle Source of Hawaiian Shield Basalts. Nature, 434, 590-597.

https://doi.org/10.1038/nature03411

[11] Sobolev, A.V., Hofmann, A.W., Kuzmin, D.V., Yaxley, G.M., Arndt, N.T., Chung, S.-L., et al. (2007) The Amount of Recycled Crust in Sources of Mantle-Derived Melts. Science, 316, 412-417. https://doi.org/10.1126/science. 1138113

[12] Farnetani, C.G. and Hofmann, A.W. (2009) Dynamics and Internal Structure of a Lower Mantle Plume Conduit. Earth and Planetary Science Letters, 282, 314-322. https://doi.org/10.1016/j.epsl.2009.03.035

[13] Farnetani, C.G. and Hofmann, A.W. (2010) Dynamics and Internal Structure of the Hawaiian Plume. Earth and Planetary Science Letters, 295, 231-240. https://doi.org/10.1016/j.epsl.2010.04.005

[14] Yaxley, G.M. and Green, D.H. (1998) Reactions between Eclogite and Peridotite: Mantle Refertilisation by Subduction of Oceanic Crust. Schweizerische Mineralogische und Petrographische Mitteilungen, 78, 243-255.

[15] Leitch, A.M. and Davies, G.F. (2001) Mantle Plumes and Flood Basalts: Enhanced Melting from Plume Ascent and an Eclogite Component. Journal of Geophysical Research, 106, 2047-2059. https://doi.org/10.1029/2000JB900307

[16] Lin, S.-C. and Keken, P.E. (2005) Multiple Volcanic Episodes of Flood Basalts Caused by Thermochemical Mantle Plumes. Nature, 436, 250-252. https://doi.org/10.1038/nature03697

[17] Mallik, A. and Dasgupta, R. (2012) Reaction between MORB-Eclogite Derived Melts and Fertile Peridotite and Generation of Ocean Island Basalts. Earth and Planetary Science Letters, 329-330, 97-108. https://doi.org/10.1016/j.epsl.2012.02.007

[18] Yasuda, A., Fujii, T. and Kurita, K. (1994) Melting Phase Relations of an Anhydrous Mid-Ocean Ridge Basalt from 3 to $20 \mathrm{GPa}$ : Implications for the Behavior of Subducted Oceanic Crust in the Mantle. Journal of Geophysical Research, 99, 94019414. https://doi.org/10.1029/93JB03205

[19] Takahashi, E., Shimazaki, T., Tsuzaki, Y. and Yoshida, H. (1993) Melting Study of a Peridotite KLB-1 to $6.5 \mathrm{GPa}$ and the Origin of Basaltic Magmas. Philosophical Transactions of the Royal Society of London: Series A, 342, 105-120. https://doi.org/10.1098/rsta.1993.0008

[20] Aoki, I. and Takahashi, E. (2004) Density of MORB Eclogite in the Upper Mantle. Physics of the Earth and Planetary Interiors, 143, 129-143. https://doi.org/10.1016/j.pepi.2003.10.007

[21] Basaltic Volcanism Study Project (1981) Basaltic Volcanism on the Terrestrial Planets. Pergamon Press, Inc., New York. 
Submit or recommend next manuscript to SCIRP and we will provide best service for you:

Accepting pre-submission inquiries through Email, Facebook, LinkedIn, Twitter, etc. A wide selection of journals (inclusive of 9 subjects, more than 200 journals)

Providing 24-hour high-quality service

User-friendly online submission system

Fair and swift peer-review system

Efficient typesetting and proofreading procedure

Display of the result of downloads and visits, as well as the number of cited articles Maximum dissemination of your research work

Submit your manuscript at: http://papersubmission.scirp.org/

Or contact ijg@scirp.org 